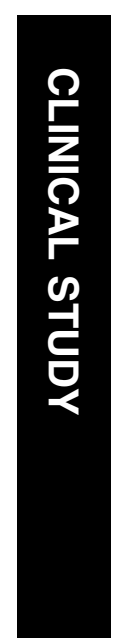

\title{
Influence of phacoemulsification surgery on progression of idiopathic epiretinal membrane
}

${ }^{1}$ Hayashi Eye Hospital,

Fukuoka, Japan

${ }^{2}$ Department of Ophthalmology, School of Medicine, Fukuoka University, Fukuoka, Japan

Correspondence: K Hayashi, Ophthalmology, Hayashi Eye Hospital, 4-7-13 Hakataekimae, Hakata-Ku, Fukuoka 812-0011, Japan Tel: + 8192431 1680; Fax: + 81924415303 E-mail: hayashi-ken@ hayashi.or.jp

Received: 28 January 2008 Accepted in revised form: 25 April 2008 Published online: 6 June 2008

\begin{abstract}
Purpose To examine whether or not phacoemulsification surgery accelerates the progression of idiopathic epiretinal membrane (ERM) by measuring the degree of ERM. Methods Forty-three consecutive eyes with idiopathic ERM that underwent phacoemulsification surgery were recruited; 41 consecutive eyes with ERM that did not undergo surgery served as controls. Foveal thickness and macular volume were measured using optical coherence tomography at 3 days after surgery (baseline), and at 3, 6, and 12 months after baseline. Visual acuity and the incidence of eyes that underwent ERM peeling surgery were also examined.

Results There were no significant differences in the foveal thickness and macular volume between the two groups at baseline. The foveal thickness gradually increased by $7.0 \%$ in the cataract surgery (CS) group by $5.3 \%$ in the no surgery group at 12 months after surgery. When comparing the groups, no significant differences were found in the foveal thickness, macular volume, or their percentage increases from baseline throughout the 12-month followup period. Only one eye in the CS group underwent ERM peeling surgery within the 12 months. Mean visual acuity did not worsen significantly in either group during follow-up. Conclusion Progression of idiopathic ERM is not accelerated by phacoemulsification surgery.
\end{abstract}

Eye (2009) 23, 774-779; doi:10.1038/eye.2008.161; published online 6 June 2008

Keywords: idiopathic epiretinal membrane; optical coherence tomography; phacoemulsification; posterior vitreous detachment
K Hayashi ${ }^{1}$ and $\mathrm{H}$ Hayashi

Introduction

It is well known that idiopathic epiretinal membrane (ERM) occurs typically after posterior vitreous detachment, and gradually progresses with aging. ${ }^{1-4}$ The ERM is also formed frequently secondary to surgery, inflammation, trauma, or tumour of the posterior segment of the eye. ${ }^{5-14}$ It is still controversial, however, as to whether or not conditions in the anterior segment of the eye contribute to the formation of ERM.

Several epidemiological studies have shown that the prevalence of ERM in patients who undergo cataract surgery (CS) is higher than that in patients who do not undergo surgery. ${ }^{15,16}$ A previous retrospective study suggested that $\mathrm{CS}$ is a major surgical cause of the occurrence of ERM. ${ }^{12}$ A recent prospective study by Jahn et $a l^{17}$ also showed that the prevalence of ERM increased up to 6 months after CS. However, as these studies were only semiquantitative and did not have controls, increases in the prevalence of ERM could not be distinguished from the natural course of ERM progression.

The purpose of the study described herein was to examine quantitatively, using optical coherence tomography (OCT), whether or not progression of idiopathic ERM is accelerated by phacoemulsification surgery. To distinguish the progression of ERM induced by phacoemulsification surgery from its natural progression, eyes with ERM that did not undergo surgery served as controls.

\section{Patients and methods}

\section{Patients}

All consecutive patients who had ERM in either eye and who were seen at the Hayashi Eye 
Hospital between January 2005 and October 2006 were screened for inclusion in this study. Preoperative exclusion criteria were pathology (other than ERM) of the retina, a history of ocular surgery or inflammation, or a pupil diameter less than $6.0 \mathrm{~mm}$ after mydriasis. Eyes scheduled for planned extracapsular cataract extraction, eyes of diabetics, eyes with any difficulty in OCT measurement or analysis, patients who refused participation, and patients who were anticipated to have any difficulties within follow-up were also excluded. Screening was continued until 45 eyes of 45 patients who were scheduled for phacoemulsification with implantation of intraocular lens (IOL), and 45 eyes of 45 patients who were not to undergo CS because of no or slight cataract were enrolled. When both eyes had ERM, only the eye with the more extensive ERM was included. The hospital Institutional Review Board approved the study protocol and informed consent was obtained from each patient.

\section{Surgical procedures}

All surgeries were performed by a single surgeon $(\mathrm{KH})$ using the same technique that has been described previously. ${ }^{18}$ First, a $3.0 \mathrm{~mm}$ scleral or $2.5 \mathrm{~mm}$ clear corneal incision was made for phacoemulsification, after which a continuous curvilinear capsulorhexis measuring about $5.0 \mathrm{~mm}$ in diameter was accomplished using a 25-gauge bent needle. After hydrodissection, endocapsular phacoemulsification of the nucleus and aspiration of the residual cortex were carried out. Using a steel keratome, the wound was then enlarged to a width of between 3.0 and $4.1 \mathrm{~mm}$ for IOL implantation. The lens capsule was inflated with sodium hyaluronate $1 \%$ (Healon; AMO, Santa Ana, California, USA), and the IOL was inserted into the capsular bag using folding forceps or an injector. After IOL insertion, the viscoelastic material was thoroughly evacuated. All surgeries included in the analysis were uneventful and the IOLs were implanted accurately within the capsular bag.

\section{Main outcome measures}

The central retinal (foveal) thickness and macular volume of all patients were measured using the Stratus OCT (OCT-3; Carl Zeiss Meditec, Dublin, California, USA) at 3 days after CS or on the day when the diagnosis of ERM was made in eyes that did not undergo CS (baseline), and at 3, 6, and 12 months after baseline. ${ }^{19}$ As several studies showed that cataract influences on the retinal thickness measurement of OCT, we performed the baseline examination at 3 days after surgery, and the data at 3 days after surgery were regarded to be almost the same as preoperative value. ${ }^{20,21}$ Best-corrected visual acuity and intraocular pressure (IOP) were also tested at each examination. Best-corrected visual acuity on decimal charts was converted to the logarithm of minimal angle of resolution (logMAR) scale for statistical analysis. The objective refractive status and keratometric cylinder were examined using an autokeratorefractometer (KR-7100; Topcon. Tokyo, Japan). Firmness of the nucleus was graded by the surgeon from grade 1 to 5, according to the Emery-Little classification. The duration of ultrasound (US) emission, the average US power emitted, and the total volume of irrigating solution used were estimated during surgery. The sum of the duration and average US power was defined as the total US energy. Flare intensity was measured at approximately 7 days after surgery using the flare metre (FC-1000; Kowa, Tokyo, Japan). All measurements were performed by ophthalmic technicians who were unaware of the purpose of this study.

During the OCT procedure, each eye underwent six radial scans centred on the fovea. Each radial scan line consisted of 100 samples, each of which was $5.92 \mathrm{~mm}$ in length, yielding a total of 600 samples from the six radial scans. Macular volume was calculated as follows. A central macular thickness map measuring $3.45 \mathrm{~mm}$ in diameter was generated; the diameters of the middle and inner circles were 2.22 and $1.00 \mathrm{~mm}$, respectively. The circular map was then subdivided into nine quadrants, and mean retinal thickness was calculated for each of the nine quadrants from the previously obtained radial scans. Multiplying the mean retinal thickness by the area of the quadrant generated the volume for each of the nine quadrants, and the total macular volume was thus determined as the sum of the volume of the nine quadrants.

\section{Statistical analysis}

Differences between the CS group and the no surgery (NS) group in foveal thickness, macular volume, visual acuity, IOP, manifest spherical equivalent value, keratometric cylinder, and other continuous variables were compared using the Mann-Whitney $U$-test. This test was used because the sample size was different between the two groups. The ratio of male and female, the ratio of left to right eyes, and other discrete variables were compared using the Fisher exact probability test. A $P$-value $\leqslant 0.05$ was considered to be statistically significant.

\section{Results}

Of the 90 patients initially enrolled, six were excluded from the analysis: two were hospitalized, one had moved from the area, two did not appear for a follow-up 
examination because of a scheduling conflict, and one refused the OCT examination. Accordingly, 43 eyes of 43 patients in the CS group and 41 eyes of 41 patients completed the examinations and remained in the analysis. The average age of the patients ( \pm SD) was $64.9 \pm 7.0$ years, with a range of $42-79$ years; there were 27 men and 57 women. No statistically significant differences were found between the CS and NS groups regarding age (65.7 vs 64.1 years; $P=0.3141$ ), the ratio of men to women $(12 / 31$ vs $15 / 26 ; P=0.3946)$, ratio of left to right eyes $(18 / 25$ vs $22 / 19 ; P=0.2791)$, manifest spherical equivalent ( $-0.41 \mathrm{D}$ vs $-1.65 \mathrm{D} ; P=0.6643)$, or keratometric cylinder $(0.75 \mathrm{D}$ vs $0.73 \mathrm{D} ; P=0.8580)$.

The mean grade of nuclear firmness was $2.1 \pm 0.7$. The mean value of the average US power, the duration of US emission, and the total volume of irrigating solution were $32.5 \pm 5.9 \%, 42.5 \pm 16.1 \mathrm{~s}$, and $70.0 \pm 32.9 \mathrm{ml}$, respectively. The mean total US energy was not correlated significantly with flare intensity $(P=0.3538)$, foveal thickness $(P \geqslant 0.2401)$, macular volume $(P \geqslant 4047)$, or the percent increases from baseline $(P \geqslant 0.2225)$.

Figures 1 and 2 show changes in the foveal thickness and macular volume, respectively. There was no significant difference between the CS and NS groups in the foveal thickness or macular volume at baseline. The foveal thickness gradually increased by $7.0 \%$ in the CS group and by $5.3 \%$ in the NS group at 12 months after baseline. When comparing the two groups, no significant differences were found in either the foveal thickness or macular volume throughout the follow-up period. There were also no significant differences in the percent increases in either the foveal thickness or macular volume (Figure 3). Only one eye in the CS group underwent ERM peeling surgery within the 12 months follow-up period.

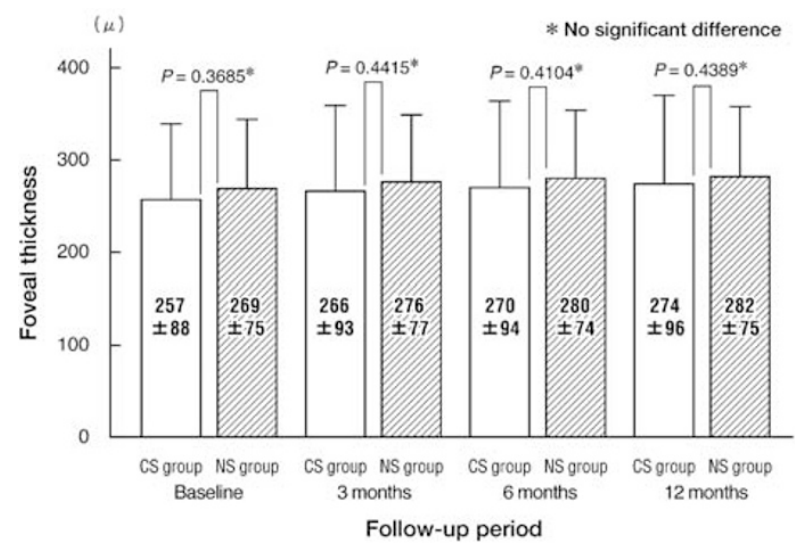

Figure 1 Comparison of the mean $( \pm S D$ ) foveal thickness between the cataract surgery (CS) and no surgery (NS) groups. No significant differences were found in the mean foveal thickness between the two groups throughout the follow-up period.
When comparing the two groups, mean visual acuity in the CS group was significantly better than that in the NS group throughout the observation period (Table 1). Mean visual acuity did not change significantly during the 12-month follow-up in either group ( $P=0.8229$ in the CS group; $P=0.9888$ in the NS group).

When comparing the two groups, the mean IOP in the CS group was significantly lower than that in the NS group at 3, 6, and 12 months after baseline (Table 1). Mean IOP did not change significantly during the followup in either group ( $P=0.3220$ in the CS group; $P=0.9119$ in the NS group).

The mean intensity of flare in the CS group was $6.3 \pm 3.1 \mathrm{mg}$ per $100 \mathrm{ml}$. No eyes showed marked increase in the flare intensity of greater than $20.0 \mathrm{mg}$ per $100 \mathrm{ml}$. No significant correlation was found between the flare

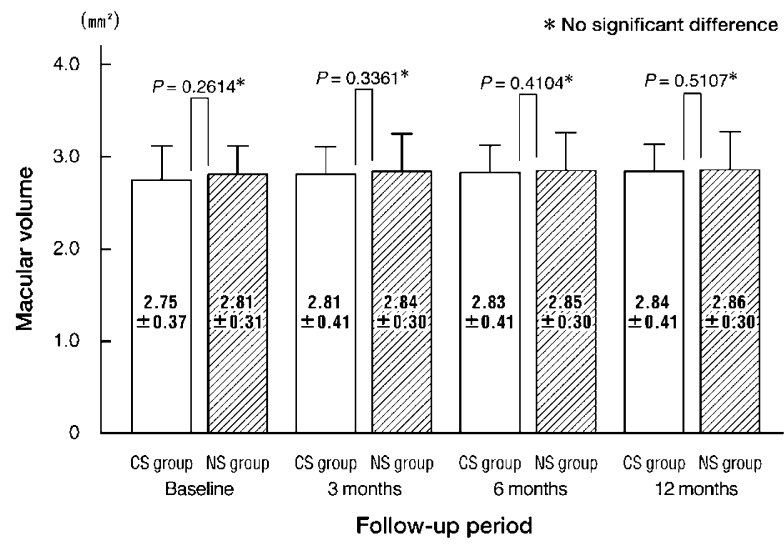

Figure 2 Comparison of mean ( $\pm \mathrm{SD}$ ) macular volume between the cataract surgery (CS) and no surgery (NS) groups. There were no significant differences in the mean macular volume between the two groups throughout the follow-up period.
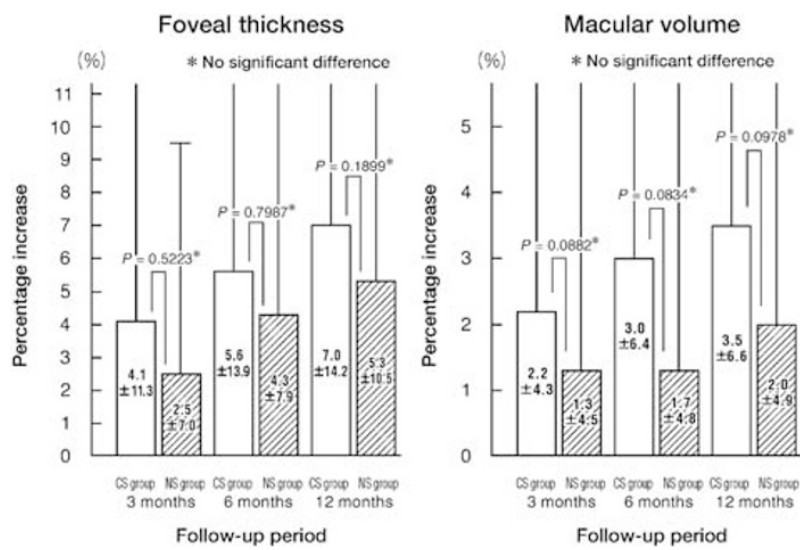

Figure 3 Comparison of mean $( \pm S D)$ percentage increase in foveal thickness and macular volume between the cataract surgery (CS) and no surgery (NS) groups. No significant differences were found between the two groups in mean percentage increase in either the foveal thickness or macular volume throughout the follow-up period. 
Table 1 Mean $( \pm S D) \log$ MAR visual acuity and intraocular pressure $(\mathrm{mmHg})$ at and after baseline in the cataract surgery (CS) and no surgery (NS) groups

\begin{tabular}{|c|c|c|c|}
\hline & $\begin{array}{c}\text { Cataract } \\
\text { surgery } \\
\text { group }\end{array}$ & $\begin{array}{c}\text { No surgery } \\
\text { group }\end{array}$ & P-value \\
\hline \multicolumn{4}{|c|}{ LogMAR visual acuity } \\
\hline Baseline & $0.05 \pm 0.09$ & $0.14 \pm 0.12$ & $>0.0001^{*}$ \\
\hline 3 months & $0.04 \pm 0.09$ & $0.15 \pm 0.12$ & $>0.0001^{*}$ \\
\hline 6 months & $0.04 \pm 0.09$ & $0.15 \pm 0.12$ & $>0.0001^{*}$ \\
\hline 12 months & $0.04 \pm 0.12$ & $0.15 \pm 0.12$ & $>0.0001^{*}$ \\
\hline \multicolumn{4}{|c|}{ Intraocular pressure $(\mathrm{mmHg})$} \\
\hline Baseline & $11.9 \pm 3.4$ & $13.0 \pm 2.8$ & $0.0596^{\dagger}$ \\
\hline 3 months & $10.9 \pm 3.5$ & $12.6 \pm 2.6$ & $0.0014^{*}$ \\
\hline 6 months & $10.7 \pm 2.4$ & $12.7 \pm 2.5$ & $0.0004^{*}$ \\
\hline 12 months & $11.3 \pm 2.8$ & $12.7 \pm 3.0$ & $0.0210^{*}$ \\
\hline
\end{tabular}

LogMAR = logarithm of minimal angle of resolution.

*Statistically significant difference.

`No significant difference. intensity and the foveal thickness $(P \geqslant 0.5475)$ or macular volume $(P \geqslant 0.4265)$, and between the flare intensity and the percent increases from the baseline $(P \geqslant 0.2054$ in the foveal thickness and $P \geqslant 0.0734$ in the macular volume).

Figure 4 shows the OCT images of representative eyes with ERM in the CS and NS groups. ERM in either eye in the CS or NS group gradually progressed slightly, but the degree of progression did not differ markedly.

\section{Discussion}

Our study demonstrated that the degree of ERM, measured as the foveal thickness and macular volume, increased gradually during 12 months in eyes that underwent phacoemulsification surgery as well as in eyes that did not undergo surgery. However, throughout this 12-month follow-up, the foveal thickness, macular volume, and the percentage increases were all similar
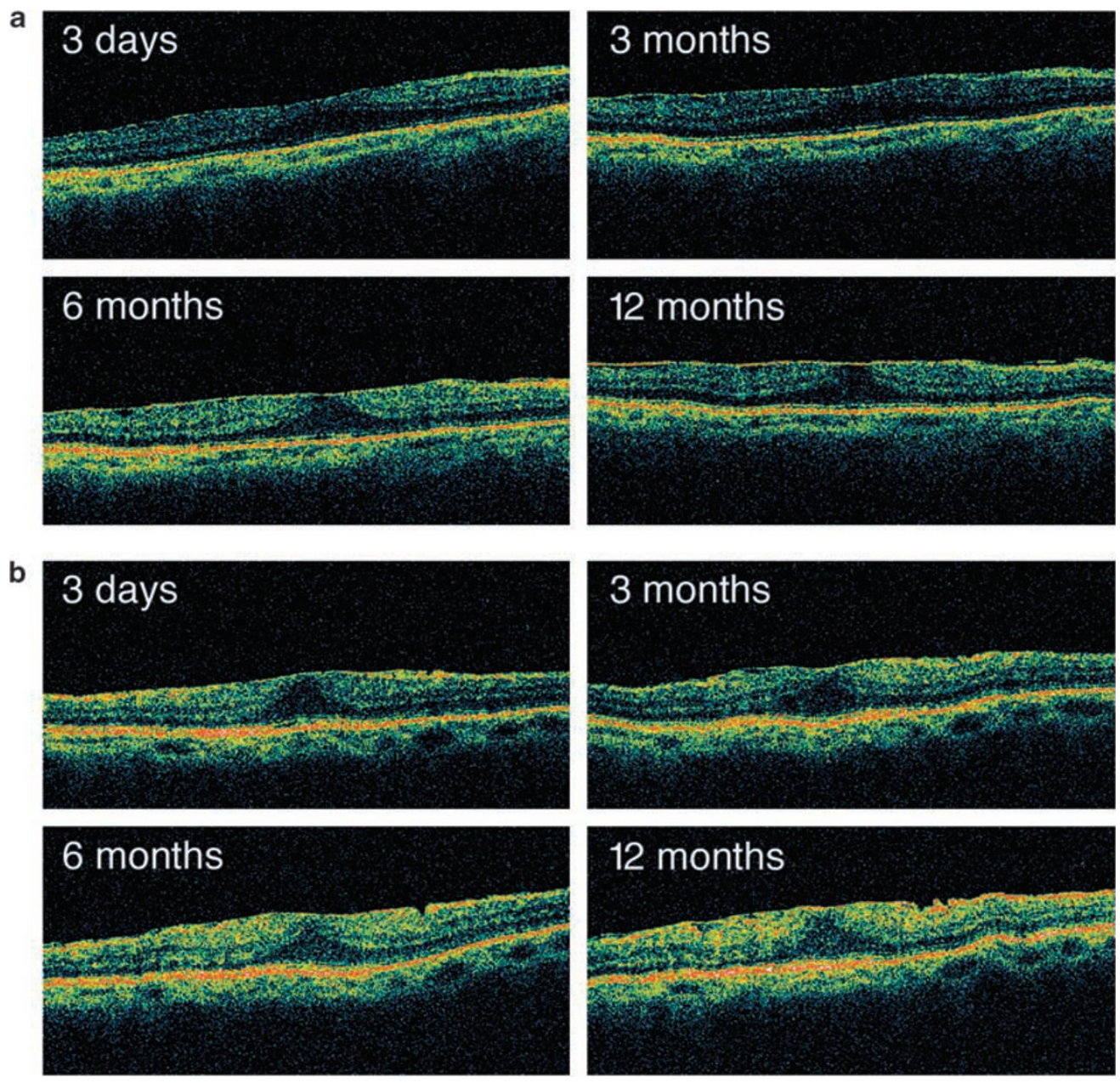

Figure 4 Changes in the optical coherence tomography of representative patients with idiopathic epiretinal membrane (ERM) in the cataract surgery (CS) (a) and no surgery (b) groups. Either eye in the CS (a) or NS (b) group showed a slight progression of ERM with time, but the difference in the progression was not marked. 
between eyes that underwent phacoemulsification surgery and those that did not undergo surgery. In addition, only one eye that had undergone phacoemulsification surgery underwent ERM peeling surgery within this period. These results indicate that the progression of ERM is not accelerated by phacoemulsification surgery.

Mean visual acuity did not worsen in eyes with or without CS within the 12-month follow-up. Although mean visual acuity in eyes that underwent surgery was better than that in eyes without surgery, the difference did not change during follow-up, suggesting that decrease in visual acuity due to progression of ERM was very slight.

Mean IOP in eyes that underwent phacoemulsification surgery was significantly lower than that in eyes without surgery. Many previous studies including ours ${ }^{22}$ showed that CS lowers the IOP for several years; ${ }^{23-25}$ this is consistent with the results obtained in this study. However, IOP in no eyes that underwent surgery was low enough to cause hypotension maculopathy, and was thus considered to be unrelated to the progression of ERM.

In complicated and difficult cases with severe postoperative inflammation, idiopathic ERM may progress markedly. However, in this series, since all surgeries were uneventful without emission of large US power, postoperative inflammation was slight. Therefore, there was no significant correlation between the flare intensity and progression of ERM as well as between the US power emitted and progression of ERM. Thus, this study, at least, indicated that ERM does not progress markedly after phacoemulsification surgery with slight inflammation.

Epidemiological studies have shown that the prevalence of ERM in patients who undergo CS is significantly higher than that in patients who did not undergo surgery. ${ }^{15,16}$ A previous retrospective study by Appiah and Hirose ${ }^{12}$ suggested that extracapsular cataract extraction is the most common surgical cause of ERM. A more recent prospective study by Jahn et al ${ }^{17}$ also showed that the prevalence of idiopathic ERM increased from $14.8 \%$ preoperatively to $25.3 \%$ at 6 months after extracapsular cataract extraction. However, these studies were only semiquantitative, and may have included both planned extracapsular cataract extraction and phacoemulsification surgery, although this point was not clarified. Furthermore, since the latter two studies did not include controls with NS, increases in the prevalence of ERM could not be distinguished from the natural course of ERM. Our quantitative study verified that the progression of idiopathic ERM after phacoemulsification surgery was virtually the same as is the natural progression of ERM.
Alternatively, progression of ERM may actually decrease in association with recent advances in CS. An explanation for this possible decrease in the progression of ERM after CS is as follows. It has been shown that an ERM occurs typically after vitreous changes including posterior vitreous detachment or retinal detachment surgery. ${ }^{1-8}$ It is reasonable to assume that planned extracapsular cataract extraction with a larger incision may cause more extensive changes to the vitreous than does phacoemulsification surgery, although controversy still remains. ${ }^{26-29}$ Specifically, modern CS is accomplished by a small incision that is less than $3.0 \mathrm{~mm}$ in length, and vitreous changes after small-incision phacoemulsification surgery are assumed to be slight, so this newer surgery may account for the recent decrease in the progression of ERM.

Several limitations exist in this study. The first is the lack of normal controls without ERM. However, in a preliminary study, the mean percentage increase in the foveal thickness of healthy eyes was $0.19 \%$ at 6 months and $-1.12 \%$ at 12 months after small-incision surgery (unpublished data). On the basis of these findings, we believe that the foveal thickness does not increase markedly after small-incision surgery in eyes without ERM. The second limitation is a possible influence of cataract on the OCT measurement. ${ }^{20,21}$ However, since only eyes with no or slight cataract were selected for this study, the influence of cataract was considered to be minimal.

In conclusion, the progression of idiopathic ERM is not accelerated by small-incision phacoemulsification surgery. Furthermore, visual acuity is not impaired markedly - at least for a year. On the basis of these results, we believe that surgeons need not hesitate to perform phacoemulsification surgery in eyes with idiopathic ERM. However, it is still unclear whether or not a secondary ERM progresses after CS in eyes with other retinal morbidity such as repaired retinal detachment or retinal pathology. Further study is called for to see if the progression of secondary ERM is accelerated or perhaps even triggered by small-incision phacoemulsification surgery in eyes with retinal pathology.

Neither author has a financial or proprietary interest in any of the materials or methods mentioned in this article and they state that the findings have not been published elsewhere.

\section{References}

1 Weise GN. Clinical features of idiopathic preretinal macular fibrosis; Schoenberg Lecture. Am J Ophthalmol 1975; 79: 349-357.

2 Hirokawa H, Jalkh AE, Takahashi M, Takahashi M, Trempe $\mathrm{CL}$, Schepens CL. Role of the vitreous in idiopathic 
preretinal macular fibrosis. Am J Ophthalmol 1986; 101: 166-169.

3 Wiznia RA. Posterior vitreous detachment and idiopathic preretinal macular gliosis. Am J Ophthalmol 1986; 102: 196-198.

4 Appiah AP, Hirose T, Kado M. A review of 324 cases of idiopathic preremacular gliosis. Am J Ophthalmol 1988; 106: 533-535.

5 Tanenbaum HL, Schepens CL, Elzeneiny I, Freeman HM. Macular pucker following retinal detachment. Arch Ophthalmol 1970; 83: 286-293.

6 Hagler WS, Aturaliya U. Macular pucker after retinal detachment surgery. Br J Ophthalmol 1971; 55: 451-457.

7 Lobes Jr LA, Burton TC. The incidence of macular pucker after retinal detachment surgery. Am J Ophthalmol 1978; 85: 72-77.

8 Kraushar MF, Morse PH. The relationship between retinal surgery and and preretinal macular fibrosis. Ophthalmic Surg 1988; 19: 843-848.

9 Hogan MJ. Inflammation and its effect on the vitreous. Trans Ophthalmol Soc UK 1975; 95: 378-381.

10 Winsthrop SR, Cleary PE, Minckler DS, Ryan SJ. Penetrating eye injuries: a histopathologic review. Br J Ophthalmol 1980; 64: 809-817.

11 Chambers RB, Mendiorvic DB, Davidorf FH. Epiretinal membranes: a clinically oriented review. Contemporary Ophthalmic Forum 1986; 101: 166-169.

12 Appiah AP, Hirose T. Secondary causes of premacular fibrosis. Ophthalmology 1989; 96: 389-392.

13 Laatikainen LI, Immonen I, Summanen P. Peripheral retinal angioma like lesion and macular pucker. Am J Ophthalmol 1989; 108: 563-566.

14 Carney MD, Jampol LM. Epiretinal membranes in sickle cell retinopathy. Arch Ophthalmol 1987; 105: 214-217.

15 Klein R, Klein BEK, Wang Q, Moss SE. The epidemiology of epiretinal membranes. Trans Am Ophthalmol Soc 1994; 92: 403-425; discussion 425-430.

16 Mitchell P, Smith W, Chey T, Wang JJ, Chang A. Prevalence and associations of epiretinal membranes: the Blue Mountains Eye Study, Australia. Ophthalmology 1997; 104: 1033-1040.

17 Jahn CE, Minich V, Moldaschel S, Stahl B, Jedelhauser P, Kremer $\mathrm{G}$ et al. Epiretinal membranes after extracapsular cataract surgery. J Cataract Refract Surg 2001; 27: 753-760.

18 Hayashi K, Hirata A, Hayashi H. Possible predisposing factors for in-the-bag and out-of-the-bag intraocular lens dislocation and outcomes of intraocular lens exchange surgery. Ophthalmology 2007; 114: 969-975.

19 Hayashi K, Hayashi H. Intravitreal vs retrobulbar injections of triamcinolone for macular edema associated with branch retinal vein occlusion. Am J Ophthalmol 2005; 139: 972-982.

20 Ching HY, Wong AC, Wong CC, Woo DC, Chan CW. Cystoid macular oedema and changes in retinal thickness after phacoemulsification with optical coherence tomography. Eye 2006; 20: 297-303.

21 van Velthoven MEJ, van der Linden MH, de Smet MD, Faber DJ, Verbraak FD. Influence of cataract on optical coherence tomography image quality and retinal thickness. Br J Ophthalmol 2006; 90: 1259-1262.

22 Hayashi K, Hayashi H, Nakao F, Hayashi F. Effect of cataract surgery on intraocular pressure control in glaucoma patients. J Cataract Refract Surg 2001; 27: 1779-1786.

23 Handa J, Henry JC, Krupin T, Keates E. Extracapsular cataract extraction with posterior chamber lens implantation in patients with glaucoma. Arch Ophthalmol 1987; 105: 765-769.

24 Shingleton BJ, Gamell LS, O'Donoghue MW, Baylus SL, King R. Long-term changes in intraocular pressure after clear corneal phacoemulsification: normal patients vs glaucoma suspect and glaucoma patients. J Cataract Refract Surg 1999; 25: 885-890.

25 Tong JT, Miller KM. Intraocular pressure change after sutureless phacoemulsification and foldable posterior chamber lens implantation. J Cataract Refract Surg 1998; 24: 256-262.

26 Tielsch JM, Legro MW, Cassard SD, Schein OD, Javitt JC, Singer AE et al. Risk factors for retinal detachment after cataract surgery. A population-based case-control study. Ophthalmology 1996; 103: 1537-1545.

27 Rowe JA, Erie JC, Baratz KH, Hodge DO, Gray DT, Butterfield L et al. Retinal detachment in Olmsted County, Minessota, 1976 through 1995. Ophthalmology 1999; 106: 154-159.

28 Igarashi C, Hayashi H, Hayashi K. Phacoemulsification after retinal detachment surgery. J Cataract Refract Surg 2004; 30: 1412-1417.

29 Kerrison JB, Marsh M, Stark WJ, Haller JA. Phacoemulsification after retinal detachment surgery. Ophthalmology 1996; 103: 216-219. 